\title{
QUANDO L'INSIEME È MEGLIO DELLA SOMMA: NANOMATERIALI IBRIDI ARGENTO-TITANIA ED ORO-TITANIA PER SENSORI ELETTROANALITICI
}

\author{
LUIGI FALCIOLA $(*)$, VALENTINA PIFFERI $(*)$, ANNA TESTOLIN $(*)$
}

Nota presentata dal s.c. Carlo Edoardo Gatti

(Adunanza del 19 dicembre 2019)

SuNTO. - L'interesse della comunità scientifica per le nanotecnologie ed i nanomateriali nasce nel 1959, in occasione della riunione annuale della American Physical Society, quando ebbe inizio un nuovo approccio con cui considerare e manipolare la materia su scala micro- e nano-metrica. Il successivo aumento dell'utilizzo di nanomateriali nella ricerca scientifica è dovuto alle loro caratteristiche peculiari: aumentato rapporto superficie/volume, nuove proprietà ottiche e fisiche, elevata superficie attiva, trasporto di materia incrementato o modificato, selettività aumentata. Tutte queste caratteristiche rendono i nanomateriali estremamente adatti per un vasto spettro di applicazioni, dall'energetica, alla catalisi ed alla sensoristica. Negli ultimi decenni, a parte l'applicazione di nanomateriali caratterizzati da un solo componente, sta rapidamente crescendo anche l'utilizzo di nanomateriali "ibridi", composti da due o più componenti in "intimo" contatto. Ciò è connesso al fatto che, poiché in un materiale ibrido l'instaurarsi di proprietà sinergiche è confinato alla regione di contatto tra i differenti componenti, nei nanomateriali, date le piccole dimensioni, questi effetti possono diventare un fattore dominante dell'intera struttura. Il risultato è appunto l'instaurazione di proprietà nuove e inaspettate (spesso volute ed interessanti) che non sono la semplice somma della proprietà dei contributi individuali. Considerato tutto ciò, i materiali ibridi sono degli ottimi candidati per applicazioni nei campi più svariati, tra i quali l'elettroanalisi, che può trarre grande vantaggio da questi sistemi. In questo contesto, questo lavoro vuole presentare la costruzione di dispositivi basati su nanomateriali ibridi metallo-semiconduttore (a base di nanoparticelle d'argento o d'oro e biossido di titanio). In questi sistemi la presenza delle nanoparticelle metalliche viene sfruttata per la determinazione elettroanalitica di ana-

(*) Gruppo di Chimica ELettroANalitica (ELAN), Dipartimento di Chimica, Università degli Studi di Milano, Italy. E-mail: luigi.falciola@unimi.it 
liti di interesse medico/diagnostico o ambientale, mentre la presenza del $\mathrm{TiO}_{2}$ risulta essenziale per superare i problemi di sporcamento e passivazione della superficie elettrodica a seguito della determinazione della molecola in esame. La possibilità di rinnovare la superficie elettrodica semplicemente irradiando il dispositivo con luce ultravioletta, permette di utilizzare i dispositivi in remoto per lungo tempo, senza il loro continuo rispristino. Nel caso del sistema $\mathrm{Ag}_{-} \mathrm{TiO}_{2}$, si è inoltre riscontrato l'instaurarsi di una interfase di "intimo contatto" tra le nanoparticelle d'argento ed il biossido di titanio, che rende l'ibrido un vero e proprio nuovo materiale, con caratteristiche differenti e con applicazioni interessanti rispetto ai suoi precursori.

$* * *$

ABSTRACT. - The interest of the scientific community for nanotechnologies and nanomaterials was born in 1959, on the occasion of the annual meeting of the American Physical Society, when a new approach began with which to consider and manipulate matter on a micro- and nano-metric scale. The subsequent increase in the use of nanomaterials in scientific research is due to their peculiar characteristics: increased surface / volume ratio, new optical and physical properties, high active surface, increased or modified material transport, increased selectivity. All these features make nanomaterials extremely suitable for a wide range of applications, from energy, to catalysis and sensors. In recent decades, apart from the application of nanomaterials characterized by a single component, the use of "hybrid" nanomaterials, composed of two or more components in "intimate" contact, is also rapidly growing. This is connected to the fact that, since in a hybrid material the establishment of synergistic properties is confined to the contact region between the different components, in nanomaterials, given the small size, these effects can become a dominant factor of the entire structure. The result is precisely the establishment of new and unexpected properties (often desired and interesting) which are not the simple sum of the ownership of individual contributions. Given all this, hybrid materials are excellent candidates for applications in the most varied fields, including electroanalysis, which can greatly benefit from these systems. In this context, this work aims to present the construction of devices based on hybrid metal-semiconductor nanomaterials (based on silver or gold nanoparticles and titanium dioxide). In these systems, the presence of metal nanoparticles is exploited for the electroanalytical determination of analytes of medical / diagnostic or environmental interest, while the presence of $\mathrm{TiO} 2$ is essential for overcoming the problems of fouling and passivation of the electrode surface following the determination of the molecule under examination. . The possibility of renewing the electrode surface simply by irradiating the device with ultraviolet light, allows you to use the devices remotely for a long time, without their continuous recovery. In the case of the $\mathrm{Ag}-\mathrm{TiO} 2$ system, an "intimate contact" interphase between the silver nanoparticles and titanium dioxide was also found, which makes the hybrid a real new material, with different characteristics and with interesting applications compared to its precursors. 


\section{INTRODUZIONE}

\subsection{Nanomateriali ed applicazioni elettroanalitiche}

L'inizio dell'era delle nanotecnologie viene fatto risalire al 29 dicembre 1959 quando, durante l'incontro annuale dell'American Physical Society, il noto fisico Richard Feynman (1918-1988), pronunciò l'ormai nota e citata frase:"There's plenty of room at the bottom" ("C'è un sacco di spazio laggiù in fondo") [1]. Con questa frase Feynman ha spianato la strada verso un nuovo approccio nella manipolazione e nel controllo delle proprietà della materia su scala miniaturizzata e quindi micro- e nano-metrica, a livello atomico e molecolare, accentuando l'attenzione sul nuovo mondo delle nanotecnologie.

Una definizione più completa e condivisa di "nanomateriale" è stata data il 18 ottobre 2011 dalla Commissione Europea: un nanomateriale è un "materiale naturale, incidentale o fabbricato contenente particelle, in uno stato non legato o come aggregato o agglomerato dove, per il 50\% o più delle particelle nella distribuzione dimensionale numerica, una o più dimensioni esterne sono comprese nel range dimensionale $1 \mathrm{~nm}$ - 100 nm". Quindi, è possibile distinguere nanoparticelle (zero dimensionali), nanofili (monodimensionali) e nanofogli (bidimensionali). Molte entità biologiche, come proteine, acidi nucleici e virus rientrano nella scala nanodimensionale, ma anche diversi materiali sintetici di varia composizione chimica, dagli elementi chimici singoli (es. metalli, carbonio, silicio) alle molecole inorganiche (es. ossidi metallici) ed organiche (es. polimeri), con numerose applicazioni scientifiche e tecnologiche, dalla sensoristica alla catalisi, grazie alle superiori proprietà ed in particolare all'elevato rapporto superficie/volume.

Nel campo specifico dell'elettrochimica e dell'elettroanalisi, la peculiarità dei nanomateriali si esprime in nuove proprietà quantomeccaniche (effetti quantici dimensionali), chimico-fisiche ed ottiche. Inoltre, i nanomateriali permettono un trasporto di materia aumentato (che facilita lo studio di processi elettrochimici veloci), hanno un'elevata superficie attiva, una migliore selettività e costi ridotti. Sono inoltre caratterizzati da un elevato rapporto segnale/rumore e da un meccanismo di diffusione migliorato o in generale modificato, passando generalmente ad una diffusione non planare (convergente o radiale). Si 
hanno inoltre diminuzione dei contributi capacitivi (la capacità del doppio strato è correlata all'area elettrodica) e riduzione della caduta ohmica (che è proporzionale alla corrente totale che passa nel circuito). La possibilità di costruire array di micro- o nano-elettrodi, permette di incrementare ulteriormente le prestazioni di questi dispositivi, che sono in grado di fornire una risposta voltammetrica di altezza (intensità di corrente) simile ai loro analoghi in dimensione macro, ma con correnti capacitive considerevolmente inferiori. [2]

Tutte queste proprietà consentono anche di fornire un maggior controllo sul microambiente locale, rendendo i nanomateriali buoni candidati per applicazioni innovative in sensori elettroanalitici. [3-4]

\subsection{Materiali ibridi ed applicazioni elettroanalitiche}

Secondo lo IUPAC (International Union of Pure and Applied Chemistry) Gold Book [5] un "ibrido" è definito come un "materiale composto da una miscela intima di componenti o inorganici o organici o di entrambi i tipi, in cui di solito tali componenti si compenetrano su scale inferiori a $1 \mu \mathrm{m}$ ". E sta proprio nel termine "intimo" che caratterizza la relazione tra i due componenti, la differenza con i materiali compositi (ossia, semplici materiali multicomponente [5]): negli ibridi, lo stretto legame ("intimo") tra i due componenti è in grado spesso di generare veri e propri nuovi materiali, con caratteristiche e proprietà differenti e molto spesso superiori rispetto ai componenti singoli. Mentre in un composito le proprietà possono essere considerate come la somma delle proprietà dei precursori, in un ibrido l'insieme risulta essere diverso e molto spesso migliore rispetto ai precursori.

In questo contesto, la chimica dei materiali ibridi rappresenta un campo molto interessante di ricerca e sviluppo, poiché permette una grande varietà di nuove applicazioni. [6]

Nei nanomateriali ibridi, definiti come la classe di materiali costituiti da due o più componenti in cui almeno uno di essi deve avere una dimensione nel regime della nanoscala [5], proprio grazie alle nanodimensioni, gli effetti sinergici causati dall'intimo contatto possono diventare un fattore dominante e caratterizzante [7].

I singoli componenti di una nanostruttura ibrida possono essere scelti tra una vasta gamma di materiali: metalli, ossidi metallici, calco- 
genuri metallici, polimeri, materiali carboniosi, ..., mentre la loro sintesi può essere fatta in diversi modi, risultando in dimensioni, forme e composizioni differenti [8]. Ad esempio, nel caso di nanoibridi metallo-metallo è possibile trovare sistemi tipo lega o core-shell, tipo Janus (ossia caratterizzati da una doppia natura), sintetizzati tramite approcci bottom-up (dal basso) o top-down (verso il basso) [9]. Per quanto riguarda altri tipi di materiali, tra gli ibridi più diffusi si possono trovare i nanomateriali organico-inorganici, dove il controllo della natura chimico-fisico e l'estensione delle interfacce ibride risultano di primaria importanza in quanto regolano proprietà quali la trasparenza, l'omogeneità chimica e la stabilità del materiale [10-13].

Una delle caratteristiche essenziali di questi ultimi materiali sono le loro dimensioni su scala nanometrica, che generano effetti di dimensione sfruttabili in fase di progettazione delle proprietà del materiale. Gli effetti del confinamento spaziale diventano sempre più importanti quando le dimensioni di un nanocristallo diminuiscono al di sotto di un certo limite critico, portando a una struttura elettronica dipendente dalla dimensione e dalla forma [10].

\subsection{Nanomateriali ibridi ed applicazioni elettroanalitiche}

Considerando le sopracitate caratteristiche e prestazioni dei materiali nanostrutturati e dei materiali ibridi, negli ultimi anni la Ricerca Scientifica si sta volgendo verso l'uso di una combinazione dei due aspetti, studiando materiali ibridi nanostrutturati, in modo da esplorare gli effetti sinergici della combinazione dei due aspetti. Tra le innumerevoli applicazioni, l'elettrochimica ed in particolar modo l'elettroanalisi possono beneficiare moltissimo di queste scoperte, permettendo di creare dispositivi a basso costo e di facile costruzione sempre più sensibili $\mathrm{e}$ con limiti di rilevabilità sempre più bassi, caratterizzati anche da alta selettività ed alcuni casi specificità, per l'analisi di sostanze anche presenti in tracce (ppm, ppb, ppt) in ambito medico/diagnostico e/o ambientale, come nella rilevazione di inquinanti emergenti. Questi dispositivi, data la loro natura miniaturizzata e a basso costo con produzione di massa, possono essere facilmente utilizzati come "sensori d'allarme", posizionati in remoto su reti idriche o su impianti industriali, permettendo un monitoraggio continuo ed affidabile, a lungo termine. 


\section{IL SISTEMA NANOIBRIDO ARGENTO-TITANIA}

Il dispositivo con nanoparticelle di argento (Fig. 1) è stato preparato deponendo uno strato di silice su vetro conduttivo tramite una procedura sol-gel. La silice è stata funzionalizzata con un silossano (APTES, (3-aminopropyl)triethoxysilane) con terminazione positiva in modo da immobilizzare le nanoparticelle di argento, cariche negativamente. Il tutto è stato ricoperto con uno strato di biossido di titanio precedentemente preparato tramite sintesi sol-gel e trattato ad alta temperatura $\left(450^{\circ} \mathrm{C}\right)$.

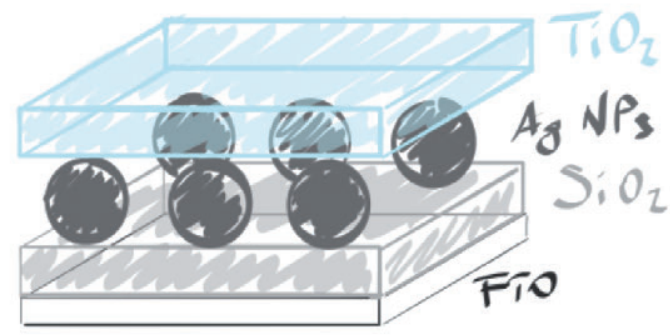

Fig. 1. Schema del dispositivo con nanoparticelle di argento.

Ogni strato del dispositivo è stato accuratamente pensato e progettato per conferire le proprietà finali desiderate. Il supporto su cui si costruisce tutto il dispositivo è un elettrodo otticamente trasparente (OTE) basato su un vetro conduttivo poiché ricoperto di un sottile strato di ossido di stagno fluorurato (FTO), resistente alla calcinazione ad alte temperature $\left(450^{\circ} \mathrm{C}\right)$. Lo strato di silice $\left(\mathrm{SiO}_{2}\right)$ viene messo perché, in combinazione con APTES, che poi è in grado di agganciare le nanoparticelle metalliche di argento (cariche negativamente) è possibile così creare un "random assembly of nanoelectrodes", ossia un assemblaggio casuale di nanoelettrodi che però risultano statisticamente ordinati e ben distanziati. Questa caratteristica presenta grandi vantaggi in applicazioni elettroanalitiche.

Lo strato finale è costituito da $\mathrm{TiO}_{2}$ (in fase anatasio, con tracce di brookite) che protegge le nanoparticelle metalliche dall'ossidazione e dal possibile rilascio in soluzione [14] e conferisce fotoattività e proprietà autopulenti al dispositivo. Maggiori dettagli sulla preparazione 
del dispositivo e sulle sue caratterizzazioni chimico-fisiche sono reperibili in [15].

Il dispositivo è stato utilizzato per la rilevazione di dopamina, norepinefrina e serotonina con limiti di rilevabilità di 0.03, 0.04 e 0.04 $\mu \mathrm{M}$ e fattori di recupero apparenti del $100-99 \%$, valori migliori di quelli richiesti dalla diagnostica clinica nelle analisi di routine [16]. Studi di interferenza hanno dimostrato il suo possibile utilizzo anche in presenza di acido ascorbico e acido urico, presenti solitamente in concentrazione da 100 a 1000 volte superiore rispetto ai neurotrasmettitori [16], anche sfruttando la differente carica elettrostatica della titania rispetto agli interferenti, all'opportuno valore di $\mathrm{pH}$. L'utilizzo con buoni risultati (LOD $=0.1 \mu \mathrm{M})$ anche in matrici reali (liquor, siero e urina), ha infine dimostrato l'estrema versatilità analitica del dispositivo. [16]

Infine, la fotorinnovabilità della superficie elettrodica del sistema, che può sporcarsi o passivarsi, a causa della matrice reale o della stessa reazione elettroanalitica che può formare sottoprodotti che si adsorbono sull'elettrodo, è stata accuratamente valutata utilizzando irraggiamenti con luce UV di diversa potenza, forma e costo. Il ripristino totale della superficie viene raggiunto dopo poco tempo di irraggiamento, aprendo la strada verso un sistema di monitoraggio integrato remoto o per analisi in flusso $[15,17]$.

Per approfondire maggiormente le ragioni delle proprietà peculiari del dispositivo, è stato condotto un lavoro concertato elettrochimico-teorico [18] che ha dimostrato che i due materiali costituenti il dispositivo $\left(\mathrm{AgNP}\right.$ e $\left.\mathrm{TiO}_{2}\right)$ sono in intimo contatto tra loro, creando una interfase in cui le proprietà elettrochimiche dell'argento vengono drasticamente modificate rispetto a quelle attese.

I risultati del doppio approccio (caratterizzazione voltammetrica combinata con spettroscopia di impedenza e calcoli DFT) hanno portato ad evidenziare come in questo sistema avvenga una donazione di elettroni dagli atomi di Ag alla titania. Gli atomi di Ag più vicini alla titania assumono una parziale carica positiva. E' possibile quindi evidenziare una vera e propria zona (quella più vicina alla titania) in cui l'elettrodo, che si ossida durante la scansione anodica del potenziale, rimane stabile nella forma ossidata (grazie alla presenza della 
titania) e non viene ridotto nella scansione inversa (Fig. 2), fino al raggiungimento di un equilibrio, in cui si osserva il ciclo ossidazione/riduzione solo per gli atomi di argento più lontani da $\mathrm{TiO}_{2}$. Per ripristinare lo strato di Ag più vicino alla titania, ossia per portarlo al suo stato di ossidazione 0 , si può operare con l'irraggiamento del dispositivo con luce UV, ottenendo il completo ripristino del picco voltammetrico (1 in Fig. $2 A$ ) posizionato a circa $0.2 \mathrm{~V}$ (SCE).

A

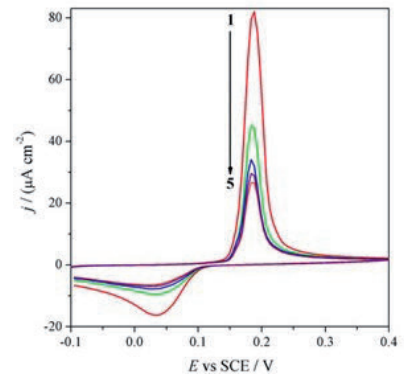

B

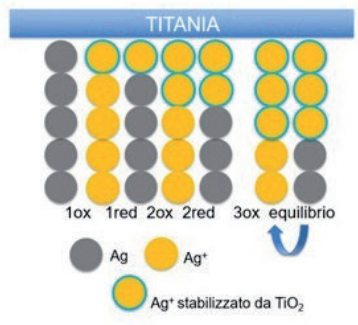

Fig. 2. A) Curve voltammetriche del dispositivo a base di $\mathrm{Ag}$, registrate a $0.1 \mathrm{~V} \mathrm{~s}^{-1}$, in $\mathrm{NaClO}_{4} 0.1 \mathrm{M}$, a temperatura ambiente e B) schema del relativo processo ciclico di ossidazione/riduzione dell'argento (stabilizzato nella sua forma ossidata alla e dalla interfase con la titania), fino al raggiungimento di un equilibrio "dinamico".

La presenza di questa interfase di intimo contatto tra $\mathrm{Ag}$ e $\mathrm{TiO}_{2}$ è anche confermata da studi di spettroscopia di impedenza in cui è necessario aggiungere nel circuito equivalente che descrive il dispositivo e interpola i dati sperimentali, un circuito RC in parallelo nel caso dell'ibrido, proprio relativo a questa interfase [18].

Questo fenomeno di trasferimento di elettroni è stato quindi ulteriormente confermato da esperimenti di fotocorrente (Fig. 3), in cui risulta evidente che, a parità di potenziale di eccitazione, l'ibrido $\mathrm{Ag} / \mathrm{TiO}_{2}$ mostra una densità di fotocorrente inferiore rispetto al solo biossido di titanio, poiché parte degli elettroni fotoprodotti vengono utilizzati per ridurre gli atomi di argento più lontani dalla titania. Questo effetto è maggiormente apprezzabile ad alti potenziali di eccitazione (es. $1 \mathrm{~V}$, SCE) dove il numero di atomi di argento ossidato è superiore, rispetto a potenziali di eccitazione più bassi (0.12 V, SCE). 
In quest'ultimo caso, solo una piccola parte di fotoelettroni viene utilizzata per la riduzione degli elettroni [18].

A

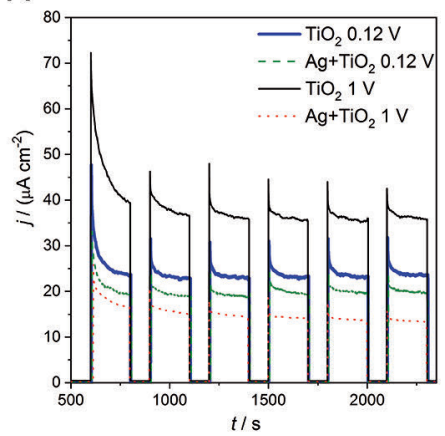

B

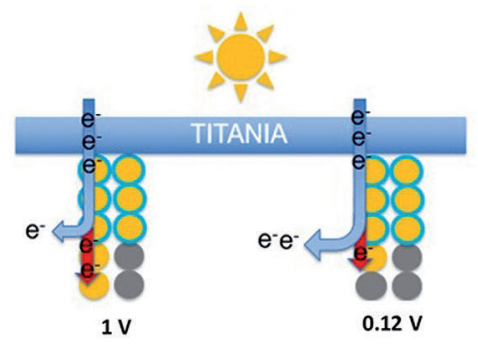

Fig. 3. A) Spettri di fotocorrente del dispositivo a base di argento, registrati a due diversi potenziali $(0.12$ e $1 \mathrm{~V}$, SCE), sotto irraggiamento con lampada UV ad alogenuro di ferro (280-400 $\mathrm{nm}$, densità di potenza effettiva di $23 \mathrm{~mW} \mathrm{~cm}^{-2}$ ) e B) schema del relativo processo di riduzione dell'argento da parte dei fotoelettroni generati all'interfase. Figura adattata da riferimento [18].

Tutte queste evidenze sperimentali e teoriche hanno permesso di fare luce sulle peculiari proprietà elettroniche di questa eterogiunzione, caratterizzata dall'intimo contatto di $\mathrm{Ag}$ e $\mathrm{TiO}_{2}$.

\section{IL SISTEMA NANOIBRIDO ORO-TITANIA}

Il dispositivo (Fig. 4) con le nanoparticelle di oro $(10 \mathrm{~nm})$ precedentemente sintetizzate con tecnica sol-gel, è stato preparato in maniera simile al precedente (vedi paragrafo 2). Le uniche differenze sono l'assenza dello strato di silice e l'aggiunta di un secondo silossano (in rapporto 36:4 con APTES) che ha permesso una funzionalizzazione diretta. Il vetro conduttivo è stato infatti direttamente funzionalizzato con l'aggiunta di un silossano con terminazione contenente zolfo (MPTMS: (3-mercaptopropyl) trimethoxysilane), che ha permesso l'immobilizzazione delle nanoparticelle d'oro, aventi grossa affinità per tale elemento. 


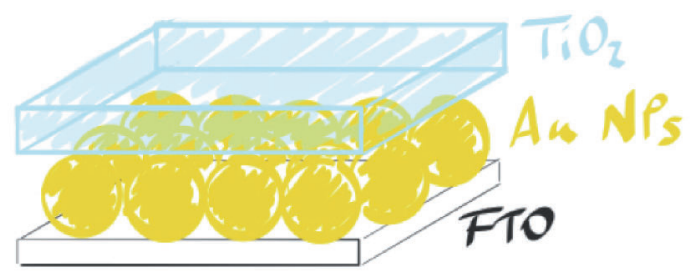

Fig. 4. Schema del dispositivo con nanoparticelle di oro.

Rispetto all'ibrido $\mathrm{Ag} / \mathrm{TiO}_{2}$, le voltammetrie cicliche (Fig. 5A) del dispositivo $\mathrm{Au} / \mathrm{TiO}_{2}$, mostrano una maggiore somiglianza con quelle del supporto (FTO) modificato con le sole nanoparticelle di Au, evidenziando come le proprietà intrinseche dell'oro siano preservate. Inoltre, l'area del picco di ossidazione e quella di riduzione sono simile e non sbilanciate come nel caso precedente. La diversa forma del plateau di ossidazione potrebbe essere dovuta a una diversa disposizione delle nanoparticelle d'oro, che nel caso del dispositivo sono meno aggregate e "più protette" dalla presenza dello strato di titania protettivo. Si ipotizza pertanto che in questa struttura non avvenga alcun trasferimento di carica tra i due materiali del composito, come anche confermato dagli esperimenti di fotocorrente (Fig. 5B).

A

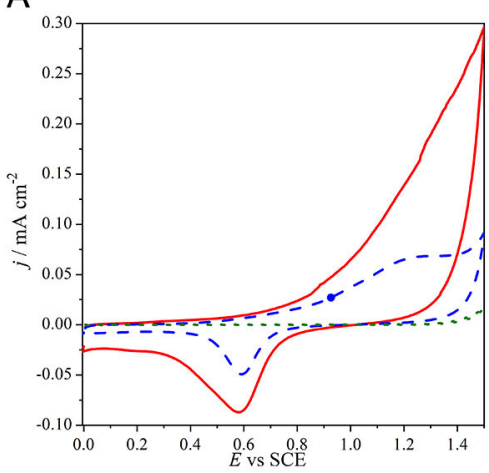

B

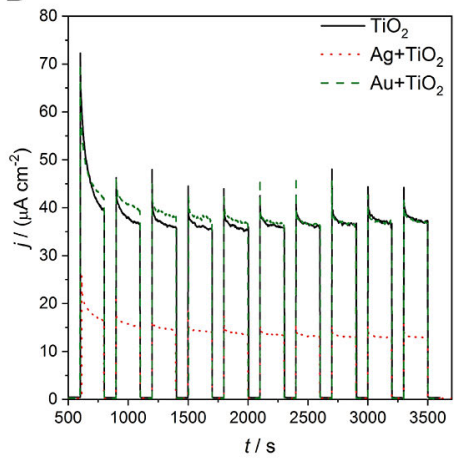

Fig. 5. A) Curve voltammetriche del dispositivo a base di Au (curva blu tratteggiata), di AuNPs (curva rossa continua), di $\mathrm{TiO}_{2}$ (curva verde, tratto piccolo), registrate a $0.1 \mathrm{~V} \mathrm{~s}^{-}$ 1, in $\mathrm{NaClO}_{4} 0.1 \mathrm{M}$, a temperatura ambiente e B) spettri di fotocorrente del dispositivo a base di oro (paragonato con quello a base di argento), registrati a $1 \mathrm{~V}$ (SCE), sotto irraggiamento con lampada UV ad alogenuro di ferro (280-400 nm, densità di potenza effettiva di $23 \mathrm{~mW} \mathrm{~cm}^{-2}$ ). 
Librido e il $\mathrm{TiO}_{2}$ semplice mostrano la stessa densità di fotocorrente, il che significa che nel caso dell'ibrido tutti gli elettroni coinvolti sono utilizzati per la generazione della fotocorrente anodica e non è quindi presente alcuna donazione elettronica dall'oro alla titania. Anche gli esperimenti di spettroscopia di impedenza (Fig. 6A) evidenziano come l'ibrido sia più simile al sistema con le sole nanoparticelle d'oro, che non si stanno adattando alla titania come precedentemente osservato nel caso dell'argento. I circuiti equivalenti utilizzati per interpolare i dati sperimentali (Fig. 6B), non presentano infine, il terzo circuito RC parallelo, necessario nel dispositivo precedente, denotando l'assenza dell'interfase metallo/titania nel caso dell'oro.

A

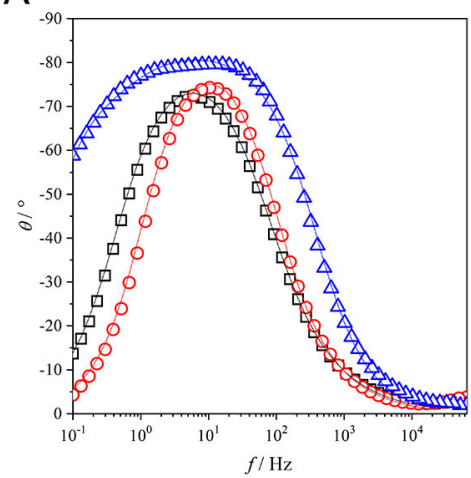

B

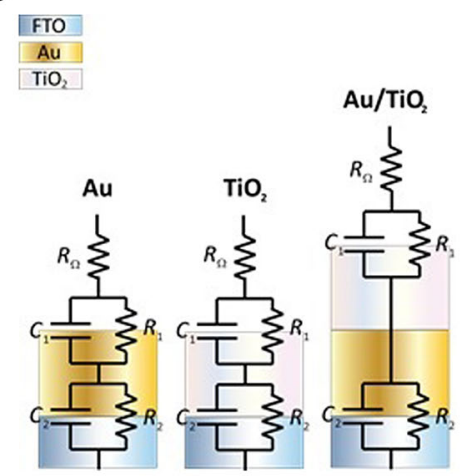

Fig. 6. A) Curve di impedenza (Bode Plot) del dispositivo a base di Au (curva rossa a pallini), di $\mathrm{AuNPs}$ (curva nera a quadretti), di $\mathrm{TiO}_{2}$ (curva blu a triangoli), registrati a - $0.1 \mathrm{~V}(\mathrm{SCE})$ e B) relativi circuiti equivalenti per il fitting (linee continue) delle curve sperimentali.

\subsection{Applicazioni elettroanalitiche del dispositivo $\mathrm{Au} / \mathrm{TiO}_{2}$}

Anche questo secondo dispositivo a base di nanoparticelle di oro è stato applicato nella rilevazione elettroanalitica di Diclofenac (2- [2(2,6-dichloroanilino) fenil] acido acetico), un farmaco antinfiammatorio non steroideo ampiamente usato per il trattamento del dolore e delle malattie infiammatorie. Decimo farmaco più venduto in Italia nel 2018, è classificato come contaminante emergente e nel 2013 è stato inserito, insieme ad altri prodotti farmaceutici, in un elenco di controllo 
da parte dell'Unione Europea nella "Direttiva quadro sulle acque europee”, contenente tutti quei composti emergenti che dovrebbero essere dannosi per l'ambiente. Secondo questo documento, è stato fissato il seguente standard di qualità ambientale per Diclofenac: le concentrazioni massime consentite sono di 0,1 g/ L in acque dolci e 0,01 mg / L in acque marine (2013/39 / CE, Direttiva Europea, Diclofenac EQS Dossier 2011). La presenza di diclofenac nell'ambiente è principalmente dovuta alla sua escrezione, insieme ai suoi metaboliti, da parte di esseri umani e animali. Questi composti raggiungono l'impianto di trattamento delle acque reflue dove possono seguire due percorsi diversi: (i) da un lato, parte di Diclofenac viene assorbita dai fanghi che finendo in discarica, permettono a Diclofenac di entrare nelle acque superficiali per percolazione; (ii) dall'altro lato, Diclofenac rimane nelle acque reflue e viene scaricato direttamente nelle acque superficiali. Pertanto, anche i sistemi convenzionali di trattamento delle acque reflue non sono in grado di abbattere la presenza di questo inquinante con una buona percentuale di rimozione $[19,20]$.

Sfruttando l'affinità delle nanoparticlle di Au per Diclofenac [21], abbiamo testato la capacità del dispositivo di rilevare questa molecola, sfruttando poi la presenza di $\mathrm{TiO}_{2}$, al fine di rinnovare la superficie del dispositivo dopo l'analisi, che generalmente porta alla formazione di sottoprodotti che sporcano l'elettrodo, rendendolo inutilizzabile per le rilevazioni successive. $[15,16,21]$

In particolare, in Fig. 7, viene mostrato il picco voltammetrico per la rilevazione di Diclofenac ad una concentrazione di $710^{-5} \mathrm{M}$, in tampone fosfato a $\mathrm{pH}=4$. Dopo la determinazione dell'analita, l'elettrodo si sporca, a causa dei sottoprodotti della reazione elettrodica durante la rilevazione [21, 22]. Tale sporcamento non è risolvibile per semplice lavaggio dell'elettrodo, come dimostra la permanenza del picco voltammetrico dopo il lavaggio con acqua.

Solo dopo trattamento con luce UV (irraggiamento di un'ora con lampada UV a $365 \mathrm{~nm}$ posta a $20 \mathrm{~cm}$ dal supporto, anche in presenza della soluzione e del vetro del contenitore in cui è alloggiato l'elettrodo), la situazione elettrodica originaria viene ripristinata. Anche l'utilizzo di un'altra fonte luminosa più facilmente reperibile e maneggiabile (LED, con una lunghezza d'onda di emissione a $390 \mathrm{~nm}$ ), ha portato al 
perfetto recupero della superficie elettrodica, confermando l'applicabilità di questa procedura per il mantenimento in perfetta pulizia dell'elettrodo di lavoro ed aprendo quindi la strada per applicazioni in monitoraggio continuo e remoto.

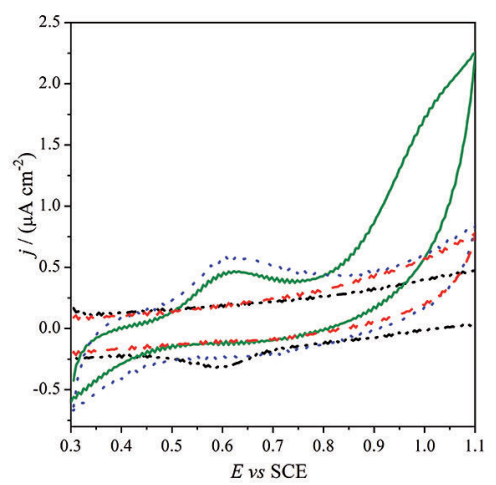

Fig. 7. Curve voltammetriche del dispositivo a base di oro, nelle varie fasi di "sporcamento" e "pulizia": prima registrazione in assenza di analita (curva nera tratto-punto); registrazione in presenza di Diclofenac $710^{-5} \mathrm{M}$ (curva verde continua); registrazione dopo lavaggio del dispositivo per semplice risciacquo in acqua (curva blu a punti); registrazione dopo recupero fotocatalitico sotto irraggiamento per un'ora (lampada UV con $(\max )=365 \mathrm{~nm}$ ) (curva rossa tratteggiata). Curve registrate a $0.1 \mathrm{~V} \mathrm{~s}^{-1}$, in tampone fosfato a $\mathrm{pH}=4$, a temperatura ambiente.

\section{CONCLUSIONI}

L'uso di nanomateriali ibridi, caratterizzato da comportamenti e caratteristiche molto interessanti, ha aperto la strada verso applicazioni promettenti in molti campi, tra cui l'elettroanalisi, che si sta molto avvantaggiando di questi sistemi per la realizzazione di dispositivi sensoristici caratterizzati da prestazioni elevate.

Le nano-eterogiunzioni, che costituiscono il cuore di questi siste$\mathrm{mi}$, se opportunamente progettate, permettono di migliorare le funzionalità sinergiche e consentire di ottenere "nuovi materiali " caratterizzati da proprietà chimico-fisiche macroscopiche che non sono semplicemente la somma di quella dei rispettivi precursori, ma vanno oltre il semplice materiale. 
Tuttavia, la ricerca su tali materiali è spesso dominata da procedure di prova ed errore, mentre risulta essenziale una profonda comprensione atomistica dei fenomeni all'interno della regione di giunzione, guidata da solide evidenze sperimentali tratte da tecniche e procedure diverse, per giungere ad una progettazione appropriata del dispositivo finale.

Questo è l'approccio adottato nel lavoro qui presentato, che può o meglio dovrebbe essere utilizzato anche in altri casi dove il contributo delle eterogiunzioni risulta cruciale.

\section{RINGRAZIAMENTI}

In memoria del caro Professor Torquato Mussini.

\section{BIBLIOGRAFIA}

1. Feynman, R. P. Caltech Engineering and Science. 23:5. 1960, pp 22-36

2. Compton, R. G., Banks, C. E. Understanding Voltammetry; Imperial College Press, London, 2001.

3. Welch, C. M., Compton, R. G. The Use of Nanoparticles in Electroanalysis: A Review. Anal. Bioanal. Chem. 2006, 384, 601-619.

4. Campbell, F. W., Compton, R. G. The Use of Nanoparticles in Electroanalysis: An Updated Review. Anal. Bioanal. Chem. 2010, 396 (1), 241-259.

5. PAC, 2007, 79, 1801. (Definitions of terms relating to the structure and processing of sols, gels, networks, and inorganic-organic hybrid materials (IUPAC Recommendations 2007)) on page 1811

6. Sanchez, C., Belleville, P., Popalld, M., Lionel, N. Applications of Advanced Hybrid Organic-Inorganic Nanomaterials: From Laboratory to Market. Chem. Soc. Rev. 2011, 40 (2), 588-595.

7. Costi, R., Saunders, A. E., Banin, U. Colloidal Hybrid Nanostructures: A New Type of Functional Materials. Angew. Chemie - Int. Ed. 2010, 49 (29), 48784897

8. Huang, X., Tan, C., Yin, Z., Zhang, H. 25th Anniversary Article: Hybrid Nanostructures Based on Two-Dimensional Nanomaterials. Adv. Mater. 2014, 26 (14), 2185-2203.

9. Toshima, N., Yonezawa, T. Bimetallic Nanoparticles: Novel Materials for Chemical and Physical Applications. 1998, 1179-1201.

10. Donegá, C. D. M. Synthesis and Properties of Colloidal Heteronanocrystals. Chem. Soc. Rev. 2011, 40 (3), 1512-1546. 
11. Ingrosso, C., Bianco, G. V., Corricelli, M., Comparelli, R., Altamura, D., Agostiano, A., Striccoli, M., Losurdo, M., Curri, M. L., Bruno, G. Photoactive Hybrid Material Based on Pyrene Functionalized PbS Nanocrystals Decorating CVD Monolayer Graphene. ACS Appl. Mater. Interfaces 2015, 7 (7), 4151-4159.

12. Corricelli, M., Altamura, D., De Caro, L., Guagliardi, A., Falqui, A., Genovese, A., Agostiano, A., Giannini, C., Striccoli, M., Curri, M. L. Self-Organization of Mono- and Bi-Modal PbS Nanocrystal Populations in Superlattices. CrystEngComm 2011, 13 (12), 3988-3997.

13. Ingrosso, C., Corricelli, M., Bettazzi, F., Konstantinidou, E., Bianco, G. V., Depalo, N., Striccoli, M., Agostiano, A., Curri, M. L., Palchetti, I. Au Nanoparticle: In Situ Decorated RGO Nanocomposites for Highly Sensitive Electrochemical Genosensors. J. Mater. Chem. B 2019, 7 (5), 768-777.

14. Cloake, S.J., Toh, H.S., Lee, P.T., Salter, C., Johnston, C., Compton, R.G. Anodic stripping voltammetry of silver nanoparticles: Aggregation leads to incomplete stripping. Chemistry Open 2015, 4(1), 22-26.

15. Soliveri, G., Pifferi, V., Panzarasa, G., Ardizzone, S., Cappelletti, G., Meroni, D., Sparnacci, K., Falciola, L. Self-Cleaning Properties in Engineered Sensors for Dopamine Electroanalytical Detection. Analyst 2015, 140 (5), 1486-1494.

16. Pifferi, V., Soliveri, G., Panzarasa, G., Cappelletti, G., Meroni, D., Falciola, L. Photo-Renewable Electroanalytical Sensor for Neurotransmitters Detection in Body Fluid Mimics. Anal. Bioanal. Chem. 2016, 408 (26), 7339-7349.

17. Pifferi, V., Soliveri, G., Panzarasa,G., Ardizzone, S., Cappelletti, G., Meroni, D., Falciola, L. Electrochemical sensors cleaned by light: a proof of concept for on site applications towards integrated monitoring systems. RSC Advances 2015, 5 , 71210-71214.

18. Di Liberto, G., Pifferi, V., Lo Presti, L., Ceotto, M., Falciola, L. Atomistic Explanation for Interlayer Charge Transfer in Metal-Semiconductor Nanocomposites: The Case of Silver and Anatase. J. Phys. Chem. Lett. 2017, 8 (21), 5372-5377.

19. Lonappan, L., Brar, S. K., Das, R. K., Verma, M., Surampalli, R. Y. Diclofenac and Its Transformation Products: Environmental Occurrence and Toxicity - A Review. Environ. Int. 2016, 96, 127-138.

20. Mohapatra, D. P., Kirpalani, D. M. Advancement in Treatment of Wastewater: Fate of Emerging Contaminants. Can. J. Chem. Eng. 2019, (October), 26212631.

21. Afkhami, A., Bahiraei, A., Madrakian, T. Gold Nanoparticle/Multi-Walled Carbon Nanotube Modified Glassy Carbon Electrode as a Sensitive Voltammetric Sensor for the Determination of Diclofenac Sodium. Mater. Sci. Eng. C 2016, 59, 168-176.

22. Yang, X., Wang, F., Hu, S. Enhanced Oxidation of Diclofenac Sodium at a Nano-Structured Electrochemical Sensing Film Constructed by Multi-Wall Carbon Nanotubes-Surfactant Composite. Mater. Sci. Eng. C 2008, 28 (1), 188 194. 
